\title{
ON A NONHOMOGENEOUS SYSTEM OF PRESSURELESS FLOW
}

BY

YI DING (Department of Mathematics, New Jersey City University)

AND

FEIMIN HUANG (Institute of Applied Mathematics, AMSS, Academia Sinica, China)

Abstract. In this paper, a nonhomogeneous system of pressureless flow

$$
\rho_{t}+(\rho u)_{x}=0, \quad(\rho u)_{t}+\left(\rho u^{2}\right)_{x}=\rho x
$$

is investigated. It is found that there exists a generalized variational principle from which the weak solution is explicitly constructed by using the initial data; i.e.,

$$
\rho(x, t)=-\frac{\partial^{2}}{\partial x^{2}} \min _{y} F(y ; x, t), \quad \rho(x, t) u(x, t)=\frac{\partial^{2}}{\partial x \partial t} \min _{y} F(y ; x, t)
$$

hold in the sense of distributions, where $F(y ; x, t)$ is a functional depending on the initial data. The weak solution is unique under an Oleinik-type entropy condition when the initial data is of measurable function. It is further shown that the solution $u(x, t)$ converges to $x$ as $t$ tends to infinity. The proofs are based on the generalized variational principle and careful studies on the generalized characteristics introduced by Dafermos $[5]$.

1. Introduction. In this paper, we consider a nonhomogeneous system of pressureless flow

with the initial data

$$
\left\{\begin{array}{l}
\rho_{t}+(\rho u)_{x}=0, \\
(\rho u)_{t}+\left(\rho u^{2}\right)_{x}=\rho x
\end{array}\right.
$$

$$
\rho_{0}(x) \in L_{l o c}^{1}(R), \quad u_{0}(x) \in L^{\infty}(R), \quad \rho_{0}(x)>0, \text { a.e. }
$$

Here $\rho$ and $u$ denote the density of mass and velocity respectively and $\rho x$ should be considered as the external force.

Recently, the problem of pressureless type system has attracted a great deal of attention since $[1,4,9]$. We refer to $[1,3,4,9,11,12,15,16]$ and references therein. The main

Received February 3, 2003.

2000 Mathematics Subject Classification. Primary 35L65, 82C21.

Key words and phrases. Nonhomogeneous system of pressureless flow, Generalized variational principle, Lebesgue-Stieltjes integral.

E-mail address: yding@njcu.edu

E-mail address: fhuang@mail.amt.ac.cn 
feature of such a system is the formulation of the delta wave, no matter how smooth the initial data are. This poses new challenges to the analysis of the solutions. An interesting phenomena is that a generalized variational principle (GVP) exists for the homogeneous pressureless flow (cf $[9,11,15]$ ). By the procedure of GVP, the measured value solution can be explicitly constructed using the initial data, although the pressureless flow is essentially a coupled system. It is worthwhile to point out that neither the usual Lax nor Oleinik entropy condition is enough to ensure the uniqueness of weak solution under the usual initial condition. An additional initial condition, called the energy condition (see [11]), needs to be imposed, which together with the Oleinik entropy condition, guarantee the well-posedness for the homogeneous pressureless flow.

In this paper we consider a nonhomogeneous system of pressureless flow (1.1) and are interested in whether the GVP still exists so that the weak solution can be exactly constructed by the initial data. We stress the fact that the velocity $u(x, t)$ is unbounded in any strip $[0, T]$ due to the effect of the nonhomogeneous term; even the initial velocity $u_{0}(x)$ is uniformly bounded and thus beyond the scope of the investigations by Oleinik [13]. This kind of nonhomogeneous system was also investigated in [7], where the solution to a nonhomogeneous Burger's equation was clearly constructed by using Hopf-Cole transformation. It is shown in [8] that the solution constructed in [7] converges to the weak solution of the corresponding Hopf's equation when the viscosity vanishes. Unlike the scalar equation of [7] and [8], our system (1.1) is a coupled system and its solution has more singularity due to the delta wave. We show in present paper that the GVP indeed exists for the nonhomogeneous system (1.1) and the weak solution is explicitly constructed by a careful study of the generalized characteristics introduced by Dafermos [5]. It is also proved that the weak solution is unique under an Oleinik-type entropy condition when the initial data is of measurable function. Compared with the homogeneous pressureless flow, we need more subtle analysis of generalized characteristics to establish the uniqueness of solution due to the fact that the back generalized characteristic is no longer a straight line. A byproduct of our result is that the solution $u(x, t)$ converges to $x$ as $t$ tends to infinity.

Before formulating our main result, we first give the definition of the entropy solution proposed by Wang, Huang, and Ding $[11,15]$. If $\rho$ and $u$ are bounded measurable functions, then $m(x, t)=\oint_{(0,0)}^{(x, t)} \rho d x-\rho u d t$ is independent of integral path and satisfies $m_{x}=\rho$ due to the fact that the first equation of (1.1) is conserved. Therefore the system (1.1) becomes, by the new variable $m(x, t)$,

$$
\left\{\begin{array}{l}
m_{t}+u m_{x}=0 \\
\left(m_{x} u\right)_{t}+\left(m_{x} u^{2}\right)_{x}=m_{x} x
\end{array}\right.
$$

We focus our attention on the system (1.3) instead of (1.1).

DEFINITION 1. Let $m(x, t)$ be of bounded variation locally in $x$ and $u(x, t)$ be bounded and measurable to $m_{x}$. Assume that the measures $m_{x}$ and $u m_{x}$ are weakly continuous in $t .(\rho, u)=\left(m_{x}, u\right)$ is called a weak solution of $(1.1)$ or $(m, u)$ is called a weak solution 
of $(1.3)$, if

$$
\left\{\begin{array}{l}
\iint \varphi_{t} m d x d t-\iint \varphi u d m d t=0 \\
\iint \psi_{t} u+\psi_{x} u^{2}+x \psi d m d t=0
\end{array}\right.
$$

holds for all $\varphi, \psi \in C_{0}^{\infty}\left(R_{+}^{2}\right)$. Here $\iint \cdots d m d t$ denotes Lebesgue-Stieltjes integral. The initial value is understood in the following sense: as $t \rightarrow 0+$,

$$
\begin{aligned}
m(x, t) & \rightarrow \int_{0}^{x} \rho_{0} d \eta, \quad \text { in } L_{l o c}^{\infty}(R), \\
\int_{0 \pm 0}^{x \pm 0} u d m & \rightarrow \int_{0}^{x} \rho_{0} u_{0} d \eta, \quad \text { in } L_{l o c}^{\infty}(R) .
\end{aligned}
$$

Definition 2. Let $(\rho, u)$ be a weak solution of $(1.1) .(\rho, u)$ is called an entropy solution of (1.1) if it satisfies an Oleinik-type entropy condition; i.e., for any $x_{1}<x_{2}$ and almost everywhere $t>0$,

$$
\frac{u\left(x_{2}, t\right)-u\left(x_{1}, t\right)}{x_{2}-x_{1}} \leq \frac{e^{t}+e^{-t}}{e^{t}-e^{-t}} .
$$

REMARK 1. The right-hand side of (1.7) is due to the effect of the nonhomogeneous term, while it is exactly $1 / t$ for the homogeneous one. It is noted that the RHS of (1.5) behaves like $1 / t$ as $t \rightarrow 0+$.

Our main result is

THEOREM 1 (Existence theorem). Let the initial data $\rho_{0}(x)$ and $u_{0}(x)$ satisfy condition (1.2). Then

$$
\begin{gathered}
\rho(x, t)=\frac{\partial m(x, t)}{\partial x}=-\frac{\partial^{2}}{\partial x^{2}} \min _{y} F(y ; x, t), \\
\rho(x, t) u(x, t)=\frac{\partial m(x, t)}{\partial x} u(x, t)=\frac{\partial^{2}}{\partial x \partial t} \min _{y} F(y ; x, t)
\end{gathered}
$$

is the entropy solution of the system (1.1), where the derivatives in (1.8) and (1.9) are understood in the sense of distributions and

$$
\begin{aligned}
F(y ; x, t) & =\int_{0}^{y} \rho_{0}(\eta)\left(\eta \operatorname{cht}+u_{0}(\eta) \operatorname{sht}-x\right) d \eta, \\
\operatorname{cht} & =\frac{e^{t}+e^{-t}}{2}, \quad \text { sht }=\frac{e^{t}-e^{-t}}{2} .
\end{aligned}
$$

Furthermore, $u(x, t)$ converges to $x$ as $t \rightarrow \infty$.

Theorem 2 (Uniqueness theorem). Let $\left(m_{1}, u_{1}\right)$ and $\left(m_{2}, u_{2}\right)$ be any two entropy solutions of (1.1) with the same initial data $\rho_{0}, u_{0}$ in the sense of Definition 2. Then

$$
m_{1}=m_{2} \quad \text { a.e., } \quad u_{1}=u_{2} \quad \text { a.e }
$$

with respect to the measure $m_{1 x}=m_{2 x}$.

REMARK 2. Our initial condition (1.5-1.6) is stronger than the usual initial condition; i.e., as $t \rightarrow+0$, the measures $\rho$ and $\rho u$ weakly converge to $\rho_{0}, \rho_{0} u_{0}$ respectively. This is 
because our initial data is of measurable function, not Radon measure. It is noted that the solution constructed in Theorem 1 satisfies the initial condition (1.5-1.6) and such solution is unique in Theorem 2 .

REMARK 3. Since the solution of (1.1) is usual a Radon measure, it is natural to study the general case when the initial data is a Radon measure. We conjecture that the solution for the measure initial data can also be constructed by a similar procedure as in Theorem 1. Since the initial data is no longer a measurable function, the initial condition (1.5-1.6) is too strong and the usual initial condition should be introduced instead. Similar to the homogeneous pressureless flow, we guess that the Oleinik-type entropy condition (1.7) and an energy condition introduced by [11] (i.e., the measure $\rho u^{2}$ weakly converges to $\rho_{0} u_{0}^{2}$ as $t \rightarrow 0$ ) are enough to guarantee the uniqueness for the nonhomogeneous system (1.1). This will be investigated in the future.

An outline of this paper is as follows. In Sec. 2, we introduce the GVP and construct an entropy solution using the initial data. In Sec. 3, we prove the solution constructed in Sec. 2 is indeed an entropy solution. In Sec. 4, we establish the uniqueness of the entropy solution.

2. Construction of the entropy solution. We observe that for smooth solution, the system (1.1) is equivalent to

$$
\left\{\begin{array}{l}
\rho_{t}+(\rho u)_{x}=0 \\
u_{t}+u u_{x}=x
\end{array}\right.
$$

Direct computation yields the characteristic for $(2.1)_{2}$ from the point $\left(x_{0}, 0\right)$ is

$$
x\left(x_{0}, t\right)=x_{0} \operatorname{cht}+u_{0}\left(x_{0}\right) \operatorname{sht} .
$$

We expect that the solution $u(x, t)$ of (1.1) has the similar structure as that of the scalar equation $(2.1)_{2}$. Motivated by this observation, we define for any fixed $(x, t)$,

$$
F(y ; x, t)=\int_{0}^{y} \rho_{0}(\eta)\left(\eta \operatorname{cht}+u_{0}(\eta) \operatorname{sht}-x\right) d \eta,
$$

which only depends on the initial data. Motivated by $[11,15]$, we also expect that the entropy solution of (1.1) can be constructed by a procedure of taking the minimum of $F(y ; x, t)$. To achieve this goal, we need careful studies on the functional $F(y ; x, t)$. We first have

Lemma 2.1. For any point $(x, t)$, as a function of $y, F(y ; x, t)$ has a finite low bound and achieves its minimum at some points.

Proof. Let $M=\left\|u_{0}\right\|_{L^{\infty}}$. For any $y_{1}<y_{2} \leq \frac{1}{\text { cht }}(x-M$ sht $)$, or $y_{1}>y_{2} \geq \frac{1}{\operatorname{cht}}(x+$ $M$ sht), we have

$$
F\left(y_{1} ; x, t\right)-F\left(y_{2} ; x, t\right)=\int_{y_{2}}^{y_{1}} \rho_{0}(\eta)\left(\eta \operatorname{cht}+u_{0}(\eta) \operatorname{sht}-x\right) d \eta \geq 0,
$$

which implies the Lemma. 
Let

$$
\begin{aligned}
& \nu(x, t)=\min _{y} F(y ; x, t), \quad S(x, t)=\{y ; F(y ; x, t)=\nu(x, t)\}, \\
& y_{*}(x, t)=\min _{y \in S(x, t)}\{y\}, \quad y^{*}(x, t)=\max _{y \in S(x, t)}\{y\} .
\end{aligned}
$$

It is easy to verify that

$$
\frac{1}{\operatorname{cht}}(x-M \operatorname{sht}) \leq y_{*}(x, t) \leq y^{*}(x, t) \leq \frac{1}{\operatorname{cht}}(x+M \operatorname{sht})
$$

Furthermore, we have

LEMma 2.2. $y_{*}, y^{*}$ are increasingly monotonic in $x$. In particular, $y^{*}\left(x_{1}, t\right) \leq y_{*}\left(x_{2}, t\right)$ holds for any $x_{1}<x_{2}$.

Proof. For any $y_{1} \in S\left(x_{1}, t\right), y_{2} \in S\left(x_{2}, t\right)$, we calculate

$$
F\left(y_{2} ; x_{1}, t\right)-F\left(y_{1} ; x_{1}, t\right)=\int_{y_{1}}^{y_{2}} \rho_{0}(\eta)\left(\eta \operatorname{cht}+u_{0}(\eta) \operatorname{sht}-x_{1}\right) d \eta>0
$$

and

$$
F\left(y_{1} ; x_{2}, t\right)-F\left(y_{2} ; x_{2}, t\right)=\int_{y_{2}}^{y_{1}} \rho_{0}(\eta)\left(\eta \operatorname{cht}+u_{0}(\eta) \operatorname{sht}-x_{2}\right) d \eta>0 .
$$

Combining the above two inequalities, we obtain

$$
\left(x_{2}-x_{1}\right) \int_{y_{1}}^{y_{2}} \rho_{0}(\eta) d \eta>0 .
$$

This implies $y_{2} \geq y_{1}$ and then Lemma 2.2 is proved.

LEMmA 2.3. As a function of $x$ and $t, y_{*}(x, t)$ and $y^{*}(x, t)$ are lower- and super-semicontinuous, respectively. Furthermore, at the point where $y_{*}(x, t)=y^{*}(x, t)$, both functions are continuous.

Proof. We omit the proof because it is quite similar to that of Lemma 2.2 in [15].

Since for smooth solution, the system (1.1) can be reduced to a decoupled system (2.1), the generalized characteristic method introduced by Dafermos [5] could be applied here. For each point $\left(x_{0}, t_{0}\right)$, we define the left and right backward generalized characteristics $C_{1}, C_{2}$ as follows:

$$
\begin{aligned}
& C_{1}: x=\frac{\operatorname{sh} t}{\operatorname{sh} t_{0}} x_{0}+y_{*}\left(x_{0}, t_{0}\right)\left(\operatorname{ch} t-\frac{\operatorname{sh} t}{\operatorname{sh} t_{0}} \operatorname{ch} t_{0}\right), \text { for } t<t_{0}, \\
& C_{2}: x=\frac{\operatorname{sh} t}{\operatorname{sh} t_{0}} x_{0}+y^{*}\left(x_{0}, t_{0}\right)\left(\operatorname{ch} t-\frac{\operatorname{sh} t}{\operatorname{sh} t_{0}} \operatorname{ch} t_{0}\right), \text { for } t<t_{0} .
\end{aligned}
$$

We have the following properties of the backward generalized characteristics.

LEMMA 2.4. $y_{*}(x, t)=y^{*}(x, t)$ holds along each backward generalized characteristic. Furthermore $y_{*}(x, t)=y^{*}(x, t)=y_{*}\left(x_{0}, t_{0}\right)$ along $C_{1}$ and $y_{*}(x, t)=y^{*}(x, t)=y^{*}\left(x_{0}, t_{0}\right)$ along $C_{2}$.

Proof. Here we only prove Lemma 2.4 for $C_{1}$ because the proof for $C_{2}$ is the same. Let $y_{0}=y_{*}\left(x_{0}, t_{0}\right)$. For any point $(x, t)$ located at the curve $C_{1}$ and any $y \neq y_{0}$, we 
calculate

$$
\begin{aligned}
& F(y ; x, t)-F\left(y_{0} ; x, t\right)=\int_{y_{0}}^{y} \rho_{0}(\eta)\left(\eta \operatorname{cht}+u_{0}(\eta) \operatorname{sht}-x\right) d \eta \\
= & \frac{\operatorname{sh} t}{\operatorname{sh} t_{0}} \int_{y_{0}}^{y} \rho_{0}(\eta)\left(\eta \operatorname{ch} t_{0}+u_{0}(\eta) \operatorname{sh} t_{0}-x_{0}\right) d \eta \\
& +\left(\operatorname{cht}-\frac{\operatorname{sh} t}{\operatorname{sh} t_{0}} \operatorname{ch} t_{0}\right) \int_{y_{0}}^{y} \rho_{0}(\eta)\left(\eta-y_{0}\right) d \eta .
\end{aligned}
$$

It is noted that the first term on the RHS of (2.12) is non-negative and $\operatorname{ch} t-\frac{\operatorname{sh} t}{\operatorname{sh} t_{0}} \operatorname{ch} t_{0}$ is positive. Hence $F(y ; x, t)-F\left(y_{0} ; x, t\right)>0$ if $y \neq y_{0}$ and $y_{*}(x, t)=y^{*}(x, t)=y_{0}$ holds along the curve $C_{1}$.

In terms of Lemmas 2.2-2.4, for any two backward characteristics, either they disjoin with each other except at the $x$-axis or a backward characteristic is a subset of another one. We note that for each point $\left(x_{0}, t_{0}\right)$, the backward generalized characteristics $C_{1}$, $C_{2}$, and the $X$-axis form a characteristic area. We denote it by $\Delta\left(x_{0}, t_{0}\right)$. See Fig. 2.1. Similarly, we can also show that for any two characteristic areas, either they disjoin with each other except at the $x$-axis or a characteristic area is a subset of another one. Thus, we have the following lemma.

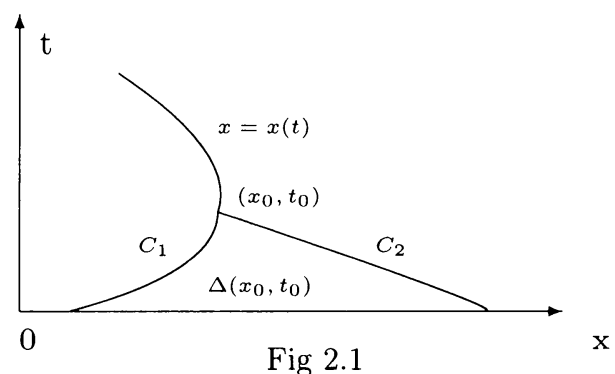

LEMMA 2.5. Each point $\left(x_{0}, t_{0}\right)$ at $t_{0}>0$ uniquely determines a Lipschitz continuous curve $x=x(t), x_{0}=x\left(t_{0}\right)$. In particular, at every $t \in\left\{\tau ; \tau \geq t_{0}\right\}$,

$\lim _{t_{2}, t_{1} \rightarrow t+0} \frac{x\left(t_{2}\right)-x\left(t_{1}\right)}{t_{2}-t_{1}}= \begin{cases}\frac{1}{\operatorname{sh} t}\left(x(t) \operatorname{ch} t-y_{*}\right), & \text { if } y_{*}=y^{*}, \\ \frac{\int_{y_{*}^{*}}^{y^{*}} \rho_{0}(\eta)\left(\eta \operatorname{sh} t+u_{0}(\eta) \operatorname{ch} t\right) d \eta}{\int_{y_{*}^{*}}^{y^{*}} \rho_{0}(\eta) d \eta}, & \text { if } y_{*} \neq y^{*}\end{cases}$

where $y_{*}=y_{*}(x(t), t)$ and $y^{*}=y^{*}(x(t), t)$.

Proof. It is observed that for any line $t=t_{1}>t_{0}$, the characteristic areas belonging to $\left(x, t_{1}\right),-\infty<x<+\infty$ disjoint each other. By this observation, we claim that all these characteristic areas cover the whole strip $0<t<t_{1}$. Indeed, let $\mathrm{A}$ be the set of points $x$ such that $\Delta\left(x, t_{1}\right)$ is located at the left side of $\left(x_{0}, t_{0}\right)$ and $\mathrm{B}$ the set of points $x$ such that $\Delta\left(x, t_{1}\right)$ is located at the right side of $\left(x_{0}, t_{0}\right)$. Let $x_{m}=\sup _{x \in A}\{x\}$ and $x_{i}=\inf _{x \in B}\{x\}$. Then we trivially have $x_{m}=x_{i}=\bar{x}$. If $\left(x_{0}, t_{0}\right) \in \Delta\left(\bar{x}, t_{1}\right)$, then our 
claim is true. If not, without loss of generality, we consider the case that $\Delta\left(\bar{x}, t_{1}\right)$ is located on the left side of $\left(x_{0}, t_{0}\right)$. By (2.10) and (2.11), there is a positive constant $\delta$ such that $y^{*}\left(\bar{x}, t_{1}\right)+\delta<y_{*}\left(y, t_{1}\right)$ holds for any $y \in B$. On the other hand, Lemma 2.3 infers that $\lim _{y \rightarrow \bar{x}} y_{*}\left(y, t_{1}\right)=y^{*}\left(\bar{x}, t_{1}\right)$. This is a contradiction.

In terms of the above argument, there is a unique point $x=x\left(t_{1}\right)$ on the line $t=t_{1}$ such that the point $\left(x_{0}, t_{0}\right) \in \Delta\left(x\left(t_{1}\right), t_{1}\right)$. Since $t_{1}>t_{0}$ is arbitrary, we obtain a curve $x(t)$ with $x_{0}=x\left(t_{0}\right)$ in $t>t_{0}$. See Fig 2.1. Furthermore, $x(t)$ is continuous due to (2.10), (2.11), and Lemmas 2.2 and 2.3. We now establish (2.13).

Let $x^{\prime}=x\left(t^{\prime}\right), x^{\prime \prime}=x\left(t^{\prime \prime}\right), t^{\prime \prime}>t^{\prime}>t$ and

$$
\begin{gathered}
y_{1}=y_{*}\left(x^{\prime}, t^{\prime}\right), \quad y^{\prime}=y^{*}\left(x^{\prime}, t^{\prime}\right), \\
y_{\prime \prime}=y_{*}\left(x^{\prime \prime}, t^{\prime \prime}\right), \quad y^{\prime \prime}=y^{*}\left(x^{\prime \prime}, t^{\prime \prime}\right) .
\end{gathered}
$$

Then Lemmas 2.2 and 2.3 imply

$$
y_{\prime \prime} \leq y_{\prime} \leq y_{*} \leq y^{*} \leq y^{\prime} \leq y^{\prime \prime}
$$

and

$$
y_{\prime \prime} \rightarrow y_{*}, \quad y^{\prime \prime} \rightarrow y^{*} \quad \text { as } \quad t^{\prime \prime} \rightarrow t
$$

We first consider the case $y_{*}=y^{*}$. Let

$$
x_{1}=\frac{\operatorname{sh} t^{\prime}}{\operatorname{sh} t^{\prime \prime}} x^{\prime \prime}+y_{\prime \prime}\left(\operatorname{ch} t^{\prime}-\frac{\operatorname{sh} t^{\prime}}{\operatorname{sh} t^{\prime \prime}} \operatorname{ch} t^{\prime \prime}\right) .
$$

Then $\left(x_{1}, t^{\prime}\right)$ is located on the left backward characteristic belonging to $\left(x^{\prime \prime}, t^{\prime \prime}\right)$ and satisfies $x_{1}<x^{\prime}$. We calculate

$$
\begin{aligned}
& \frac{x^{\prime \prime}-x^{\prime}}{t^{\prime \prime}-t^{\prime}} \leq \frac{x^{\prime \prime}-x_{1}}{t^{\prime \prime}-t^{\prime}} \\
\leq & \frac{x^{\prime \prime}}{\operatorname{sh} t^{\prime \prime}} \frac{\operatorname{sh} t^{\prime \prime}-\operatorname{sh} t^{\prime}}{t^{\prime \prime}-t^{\prime}}-\frac{y_{\prime \prime}}{\operatorname{sh} t^{\prime \prime}} \frac{\operatorname{sh} t^{\prime \prime} \operatorname{ch} t^{\prime}-\operatorname{sh} t^{\prime} \operatorname{ch} t^{\prime \prime}}{t^{\prime \prime}-t^{\prime}} .
\end{aligned}
$$

Let $t^{\prime \prime}, t^{\prime} \rightarrow t+0$; we get that the RHS of (2.19) converges to $\frac{1}{\operatorname{sh} t}\left(x(t) \operatorname{ch} t-y_{*}\right)$. Similarly we get

$$
\lim _{t^{\prime \prime}, t^{\prime} \rightarrow t+0} \frac{x^{\prime \prime}-x^{\prime}}{t^{\prime \prime}-t^{\prime}} \geq \frac{1}{\operatorname{sh} t}\left(x(t) \operatorname{ch} t-y^{*}\right) .
$$

Hence we prove $(2.13)$ for the case $y_{*}=y^{*}$.

Next we consider the case $y_{*}<y^{*}$. From the definition of $y_{*}$ and $y^{*}$, we have

$$
F\left(y^{\prime \prime} ; x^{\prime \prime}, t^{\prime \prime}\right)-F\left(y, ; x^{\prime \prime}, t^{\prime \prime}\right) \leq F\left(y^{\prime \prime} ; x^{\prime}, t^{\prime}\right)-F\left(y, ; x^{\prime}, t^{\prime}\right) \text {. }
$$

That is

$$
\int_{y,}^{y^{\prime \prime}} \rho_{0}(\eta)\left(\eta \operatorname{ch} t^{\prime \prime}+u_{0}(\eta) \operatorname{sh} t^{\prime \prime}-x^{\prime \prime}\right) d \eta \leq \int_{y_{1}}^{y^{\prime \prime}} \rho_{0}(\eta)\left(\eta \operatorname{ch} t^{\prime}+u_{0}(\eta) \operatorname{sh} t^{\prime}-x^{\prime}\right) d \eta
$$

which gives

$$
\frac{x^{\prime \prime}-x^{\prime}}{t^{\prime \prime}-t^{\prime}} \int_{y,}^{y^{\prime \prime}} \rho_{0}(\eta) d \eta \geq \int_{y,}^{y^{\prime \prime}} \rho_{0}(\eta)\left(\eta \frac{\operatorname{ch} t^{\prime \prime}-\operatorname{ch} t^{\prime}}{t^{\prime \prime}-t^{\prime}}+u_{0}(\eta) \frac{\operatorname{sh} t^{\prime \prime}-\operatorname{sh} t^{\prime}}{t^{\prime \prime}-t^{\prime}}\right) d \eta .
$$


Similarly, from the inequality

$$
F\left(y_{\prime \prime} ; x^{\prime \prime}, t^{\prime \prime}\right)-F\left(y^{\prime} ; x^{\prime \prime}, t^{\prime \prime}\right) \leq F\left(y_{\prime \prime} ; x^{\prime}, t^{\prime}\right)-F\left(y^{\prime} ; x^{\prime}, t^{\prime}\right)
$$

we obtain

$$
\frac{x^{\prime \prime}-x^{\prime}}{t^{\prime \prime}-t^{\prime}} \int_{y_{\prime \prime}}^{y^{\prime}} \rho_{0}(\eta) d \eta \leq \int_{y_{\prime \prime}}^{y^{\prime}} \rho_{0}(\eta)\left(\eta \frac{\operatorname{ch} t^{\prime \prime}-\operatorname{ch} t^{\prime}}{t^{\prime \prime}-t^{\prime}}+u_{0}(\eta) \frac{\operatorname{sh} t^{\prime \prime}-\operatorname{sh} t^{\prime}}{t^{\prime \prime}-t^{\prime}}\right) d \eta .
$$

Let $t^{\prime \prime}, t^{\prime} \rightarrow t+0$ in (2.23) and (2.25); we obtain (2.13) for the case $y_{*}<y^{*}$. Therefore Lemma 2.5 is proved.

We now construct the entropy solution of system (1.1). We define

$$
m(x, t)=\int_{0}^{y_{*}} \rho_{0} d \eta, \quad u(x, t)= \begin{cases}\frac{1}{\operatorname{sh} t}\left(x \operatorname{ch} t-y_{*}\right), & \text { if } y_{*}=y^{*}, \\ \frac{\int_{y_{*}^{*}}^{y^{*}} \rho_{0}\left(\eta \operatorname{sh} t+u_{0} \operatorname{ch} t\right) d \eta}{\int_{y_{*}}^{y^{*}} \rho_{0} d \eta}, & \text { if } y_{*} \neq y^{*} .\end{cases}
$$

By Lemma 2.2 and $(2.26)$, both $m(x, t)$ and $u(x, t)$ are of bounded variation locally in $x$. We expect that $(m(x, t), u(x, t))$ or $\left(\rho(x, t)=m(x, t)_{x}, u(x, t)\right)$ is an entropy solution of the system (1.1) in the sense of Definitions 1 and 2. This will be justified in the next section.

3. Existence of entropy solution. This section is devoted to the existence of the entropy solution. Let $\nu(x, t)$ be the minimum of the functional $F(y ; x, t)$. Then we have the following lemma.

LEMMA 3.1.

$$
\begin{gathered}
\int_{x_{1}}^{x_{2}} m(x, t) d x=\nu\left(x_{1}, t\right)-\nu\left(x_{2}, t\right), \\
\int_{t_{1}}^{t_{2}} q(x, t) d t=\nu\left(x, t_{2}\right)-\nu\left(x, t_{1}\right),
\end{gathered}
$$

where

$$
q(x, t)=\int_{0}^{y_{*}} \rho_{0}\left(\eta \operatorname{sh} t+u_{0} \operatorname{ch} t\right) d \eta
$$

Proof. The proof is based on the variational principle. We omit the proof here because it is similar to that of $[11,15]$.

From Lemma 3.1, we know that $\nu_{x}=-m, \nu_{t}=q$ holds in the sense of distributions. In order to establish $(1.4)_{1}$, we need to investigate the relation between $m$ and $q$. We have

LEMmA 3.2. $d q=u d m$ holds in the sense of Radon-Nikodym derivatives in $x$.

Proof. Without loss of generality, we assume that $y_{*}$ is not always a constant in any neighborhood of $(x, t)$. Let $x_{1}<x<x_{2}, y_{1}=y_{*}\left(x_{1}, t\right)$, and $y_{2}=y_{*}\left(x_{2}, t\right)$; then $y_{1}<y_{2}$ and

$$
y_{1} \rightarrow y_{*}(x, t), y_{2} \rightarrow y^{*}(x, t) \quad \text { as } \quad x_{1}, x_{2} \rightarrow x
$$


To prove Lemma 3.2, we only need to show

$$
\lim _{x_{2}, x_{1} \rightarrow x \pm 0} \frac{q\left(x_{2}, t\right)-q\left(x_{1}, t\right)}{m\left(x_{2}, t\right)-m\left(x_{1}, t\right)}=u(x, t) .
$$

When $y_{*}<y^{*}$, we calculate by $(3.4)$

$$
\lim _{x_{2}, x_{1} \rightarrow x \pm 0} \frac{q\left(x_{2}, t\right)-q\left(x_{1}, t\right)}{m\left(x_{2}, t\right)-m\left(x_{1}, t\right)}=\lim _{x_{2}, x_{1} \rightarrow x \pm 0} \frac{\int_{y_{1}}^{y_{2}} \rho_{0}\left(\eta \operatorname{sh} t+u_{0} \operatorname{ch} t\right) d \eta}{\int_{y_{1}}^{y_{2}} \rho_{0} d \eta}=u(x, t) .
$$

Next we consider the case $y_{*}=y^{*}$. From the definition of $y_{*}$, we have

$$
F\left(y_{2} ; x_{2}, t\right) \leq F\left(y_{1} ; x_{2}, t\right) \text {. }
$$

That is

Thus

$$
\int_{y_{1}}^{y_{2}} \rho_{0}\left(\operatorname{ch} t u_{0}+\eta \operatorname{sh} t\right) d \eta \leq \int_{y_{1}}^{y_{2}} \rho_{0} \frac{\operatorname{ch} t x_{2}-\eta}{\operatorname{sh} t} d \eta
$$

$$
\frac{q\left(x_{2}, t\right)-q\left(x_{1}, t\right)}{m\left(x_{2}, t\right)-m\left(x_{1}, t\right)} \leq \frac{\operatorname{ch} t x_{2}-y_{1}}{\operatorname{sh} t} .
$$

Let $x_{1} \rightarrow x-0, x_{2} \rightarrow x+0$. We get

$$
\lim _{x_{2}, x_{1} \rightarrow x \pm 0} \frac{q\left(x_{2}, t\right)-q\left(x_{1}, t\right)}{m\left(x_{2}, t\right)-m\left(x_{1}, t\right)} \leq u(x, t) .
$$

Similarly we have

$$
\lim _{x_{2}, x_{1} \rightarrow x \pm 0} \frac{q\left(x_{2}, t\right)-q\left(x_{1}, t\right)}{m\left(x_{2}, t\right)-m\left(x_{1}, t\right)} \geq u(x, t) .
$$

Therefore the proof of Lemma 3.2 is completed.

By Lemmas 3.1 and 3.2, the proof for $(1.4)_{1}$ becomes straightforward and will be postponed later. We now introduce another functional which is crucial to prove $(1.4)_{2}$. By the same method used in Lemma 2.5, there is a Lipschitz curve $x=X(\eta, t)$ for each point $(\eta, 0)$ on the $x$-axis except, at most, a countable number of points. The curve satisfies $\eta=X(\eta, 0)$. In terms of Lemma 2.5,

$$
\frac{\partial X(\eta, t)}{\partial t}=u(X(\eta, t), t)
$$

holds for almost every $\eta$ and $t$. For any constant $k>M$, we define

$$
\begin{gathered}
G(y ; x, t)=\int_{0}^{y} \rho_{0}(\eta)\left(\left(\eta+\eta^{2}+1\right) \operatorname{sh} t+\left(u_{0}(\eta)+k\right) \operatorname{ch} t\right)(X(\eta, t)-x) d \eta, \\
H(y ; x, t)=\int_{0}^{y} \rho_{0}(\eta)\left(\left(\eta^{2}+1\right) \operatorname{sh} t+k \operatorname{ch} t\right)(X(\eta, t)-x) d \eta .
\end{gathered}
$$

Since $X(\eta, t)$ is increasing in $\eta$ and $X(\eta, t)=x$ as $y_{*} \leq \eta \leq y^{*}$ and $\eta+\eta^{2}+1$ and $u_{0}(\eta)+k$ are positive, we easily calculate

$$
\begin{aligned}
& \mu(x, t) \triangleq \min _{y} G(y ; x, t)=G\left(y_{*} ; x, t\right), \\
& \theta(x, t) \triangleq \min _{y} H(y ; x, t)=H\left(y_{*} ; x, t\right) .
\end{aligned}
$$

Let

$$
E=\int_{0}^{y_{*}} \frac{1}{2} \rho_{0}\left(\eta \operatorname{sh} t+u_{0} \operatorname{ch} t\right) u(X(\eta, t), t) d \eta
$$


Similar to Lemma 3.1, we have

$$
\begin{aligned}
\mu_{x}=-\int_{0}^{y_{*}} & \rho_{0}(\eta)\left(\left(\eta+\eta^{2}+1\right) \operatorname{sh} t+\left(u_{0}(\eta)+k\right) \operatorname{ch} t\right) d \eta=:-\left(q+q_{1}\right) \\
\mu_{t}= & \int_{0}^{y_{*}} \rho_{0}\left(\left(\eta+\eta^{2}+1\right) \operatorname{sh} t+\left(u_{0}+k\right) \operatorname{ch} t\right) u(X(\eta, t), t) d \eta \\
& +\int_{0}^{y_{*}} \rho_{0}\left(\left(\eta+\eta^{2}+1\right) \operatorname{ch} t+\left(u_{0}+k\right) \operatorname{sh} t\right)(X(\eta, t)-x) d \eta \\
= & : 2 E+\int_{0}^{y_{*}} \rho_{0}\left(\eta \operatorname{ch} t+u_{0} \operatorname{sh} t\right)(X(\eta, t)-x) d \eta+q_{2}
\end{aligned}
$$

and

$$
\theta_{x}=-q_{1}, \quad \theta_{t}=q_{2}
$$

In terms of $(3.18)-(3.20)$, we have

$$
\kappa_{x}=-q, \quad \kappa_{t}=2 E+\int_{0}^{y_{*}} \rho_{0}\left(\eta \operatorname{ch} t+u_{0} \operatorname{sh} t\right)(X(\eta, t)-x) d \eta=: 2 E+\sigma,
$$

where

$$
\kappa(x, t) \triangleq \int_{0}^{y_{*}} \rho_{0}\left(\eta \operatorname{sh} t+u_{0} \operatorname{ch} t\right)(X(\eta, t)-x) d \eta
$$

By the same method as in (3.13)-(3.22), we get

$$
\sigma_{x}=-\int_{0}^{y_{*}} \rho_{0}\left(\eta \operatorname{ch} t+u_{0} \operatorname{sh} t\right) d \eta=:-n
$$

Proof of Theorem 1. From Lemmas 3.1 and 3.2, we know $\nu_{x}=-m(x, t), \nu_{t}=q(x, t)$, and $q_{x}=u m_{x}$. Hence

$$
\begin{aligned}
& \iint \varphi_{t} m d x d t-\iint \varphi u d m d t=\iint\left(m \varphi_{t}+q \varphi_{x}\right) d x d t \\
& =\iint\left(\nu_{t} \varphi_{x}-\nu_{x} \varphi_{t}\right) d x d t=0
\end{aligned}
$$

holds for all $\varphi \in C_{0}^{\infty}\left(R_{+}^{2}\right)$ and $(1.4)_{1}$ is proved.

On the other hand, (3.21) and (3.23) yield

$$
\iint\left(q \phi_{t}+2 E \phi_{x}+n \phi\right) d x d t=\iint\left(q \phi_{t}+(2 E+\sigma) \phi_{x}\right) d x d t=0
$$

for all $\phi \in C_{0}^{\infty}\left(R_{+}^{2}\right)$. In the same way as in Lemma 3.2 , we get $E_{x}=\frac{1}{2} u^{2} m_{x}$ and $n_{x}=x m_{x}$ in the sense of Radon-Nikodym derivatives in $x$. Thus (3.25) gives for any $\psi \in C_{0}^{\infty}\left(R_{+}^{2}\right)$,

$$
\begin{aligned}
& \iint \psi_{t} u+\psi_{x} u^{2}+x \psi d m d t \\
= & -\iint\left(q \psi_{x t}+2 E \psi_{x x}+n \psi_{x}\right) d x d t=0 .
\end{aligned}
$$

Thus $(1.4)_{2}$ is established. Since $y_{*}(x, t)$ and $y^{*}(x, t)$ converge to $x$ as $t \rightarrow 0$, the formulas of $m$ and $q$ naturally indicates the initial condition (1.5-1.6). Therefore the functions 
$(m(x, t), u(x, t))$ constructed in (2.26) is indeed a weak solution of (1.1). By (2.26) and Lemmas 3.1 and 3.2 , it is easy to check that

$$
\begin{gathered}
\rho(x, t)=\frac{\partial m(x, t)}{\partial x}=-\frac{\partial^{2}}{\partial x^{2}} \min _{y} F(y ; x, t), \\
\rho(x, t) u(x, t)=\frac{\partial m(x, t)}{\partial x} u(x, t)=\frac{\partial^{2}}{\partial x \partial t} \min _{y} F(y ; x, t) .
\end{gathered}
$$

It should be noted here that $\rho(x, t)=m(x, t)_{x}$ is usually a Radon measure.

To show the solution constructed above is an entropy solution, we only need to justify (1.7). In view of the construction of $u(x, t)$, we have

$$
u(x-0, t)=\frac{1}{\operatorname{sh} t}\left(x \operatorname{ch} t-y_{*}\right), \quad u(x+0, t)=\frac{1}{\operatorname{sh} t}\left(x \operatorname{ch} t-y^{*}\right) .
$$

On the other hand, (2.19) and (2.20) imply

$$
u(x+0, t)<u(x, t)<u(x-0, t) .
$$

Hence we calculate from (3.29) and (3.30) that

$$
\frac{u\left(x_{2}, t\right)-u\left(x_{1}, t\right)}{x_{2}-x_{1}} \leq \frac{u\left(x_{2}-0, t\right)-u\left(x_{1}+0, t\right)}{x_{2}-x_{1}} \leq \frac{\operatorname{ch} t}{\operatorname{sh} t}, \quad x_{1} \neq x_{2} .
$$

This shows that $(\rho(x, t), u(x, t))$ constructed in (3.27) and (3.28) is indeed an entropy solution of the system (1.1). Finally we point out that from $(2.6),\left|y_{*}(x, t)\right|$ and $\left|y^{*}(x, t)\right|$ are uniformly bounded as $t$ tends to infinity. Therefore (3.29) and (3.30) yield that $u(x, t) \rightarrow x$ as $t \rightarrow \infty$. Theorem 1 is proved.

4. Uniqueness of entropy solution. This section is devoted to the uniqueness of entropy solution to the nonhomogeneous system (1.1). We shall follow the idea of $[11,16]$ to establish the uniqueness Theorem 2. Our strategy is to show that any entropy solution $(\rho, u)$ coincides with the standard entropy solution constructed in Theorem 1 . This naturally leads to the uniqueness Theorem 2 .

Assume that $(\rho, u)$ or $(m, u)$ is any entropy solution in the sense of Definitions 1 and 2. Then we have

$$
m_{t}+u(x, t) m_{x}=0, \quad m_{0}(x)=\int_{0}^{x} \rho(\xi) d \xi
$$

where $u(x, t)$ satisfies

$$
\frac{u\left(x_{2}, t\right)-u\left(x_{1}, t\right)}{x_{2}-x_{1}} \leq \frac{e^{t}+e^{-t}}{e^{t}-e^{-t}}
$$

for any $x_{1}<x_{2}$ and almost all $t>0$. Let $M=\left\|u_{0}\right\|_{L^{\infty}}$ and $u_{\varepsilon}=u * j_{\varepsilon}$ with the standard mollifier $j_{\varepsilon}$; then $\left|u^{\varepsilon}\right| \leq M$ and $u_{\varepsilon x} \leq \frac{e^{t}+e^{-t}}{e^{t}-e^{-t}}$. We denote $x=X^{\varepsilon}(\xi, t)$ the characteristic curve of

$$
\left\{\begin{array}{l}
\frac{d x}{d t}=u_{\varepsilon} \\
x(0)=\xi
\end{array}\right.
$$

The characteristics $x=X^{\varepsilon}(\xi, t)$ have already been studied in [16]. We state some properties of them in the following two Lemmas. 
Lemma 4.1. (1) There exists a subsequence $X^{\varepsilon_{i}}(\xi, t)$ such that

$$
\lim _{\varepsilon_{i} \rightarrow 0} X^{\varepsilon_{i}}(\xi, t)=X(\xi, t)
$$

for all $\xi$ and $t$. Furthermore, $X(\xi, t)$ is Lipschitz continuous with respect to $t$ and is increasing with respect to $\xi$.

(2) If $X\left(\xi_{1}, t_{0}\right)=X\left(\xi_{2}, t_{0}\right)$ holds for some $\xi_{1}<\xi_{2}$ and $t_{0}>0$, then $X\left(\xi_{1}, t\right)=X\left(\xi_{2}, t\right)$ for all $t \geq t_{0}$.

(3) Let $U=\{\xi: \exists t>0$, s.t. $X(\xi-0, t) \neq X(\xi+0, t)\}$; then for any $\xi \in R / U$ and almost $t>0$,

$$
\begin{gathered}
X^{\prime}(\xi, t)=u(X(\xi, t), t), \\
u(X(\xi, t)-0, t) \leq u(X(\xi, t), t) \leq u(X(\xi, t)+0, t),
\end{gathered}
$$

where' denotes the upper derivative with respect to $t$.

(4) Let $\xi(x, t)=\sup \{\xi: X(\xi, t)<x\}, \eta(x, t)=\inf \{\xi: X(\xi, t)>x\}$; then the set $\Gamma=\{(x, t): \xi(x, t) \neq \eta(x, t), t \geq 0\}$ consists of at most countable Lipschitz continuous curves. Furthermore,

$$
\begin{gathered}
\xi(x-0, t)=\xi(x, t), \quad \eta(x, t)=\xi(x+0, t)=\eta(x+0, t), \\
X(\xi(x, t)-0, t) \leq x \leq X(\xi(x, t)+0, t) .
\end{gathered}
$$

(5) For any point $\left(x_{0}, t_{0}\right)$, there exists at least one curve $L^{\prime}$ through $\left(x_{0}, t_{0}\right)$ such that $\xi(x, t)$ keeps constant along the curve.

Lemma 4.2. If the function $u(x, t)$ is given, then the solution of Eq. (4.1) is unique. Furthermore,

$$
m(x, t)=\int_{0}^{\xi(x, t)} \rho_{0}(\xi) d \xi
$$

Proof of Theorem 2. Fix time $t$; we define

$$
C_{t}=\{x ;(x, t) \in R / \Gamma, X(\xi(x, t)-0, \tau)=X(\xi(x, t)+0, \tau), 0 \leq \tau \leq t\} .
$$

It is easy to verify that for any $0<\tau \leq t, \xi(x(\tau), \tau)$ keeps constant along the curve $x(\tau)=X(\xi(x, t), \tau)$. For any $x_{1}, x_{2} \in C_{t}$ satisfying $x_{1}<x_{2}$, let $\xi\left(x_{1}, t\right)=\xi_{1}$ and $\xi\left(x_{2}, t\right)=\xi_{2}$. Set $x_{1}(\tau)=X\left(\xi_{1}, \tau\right)$ and $x_{2}(\tau)=X\left(\xi_{2}, \tau\right), 0 \leq \tau \leq t$. Let $\phi_{1 \varepsilon}(x), \phi_{2 \varepsilon}(x) \in$ $C^{\infty}(R)$ satisfy

$$
\phi_{1 \varepsilon}(x)=\left\{\begin{array}{ll}
1, & x \leq-\varepsilon, \\
0, & x \geq 0,
\end{array} \quad \phi_{2 \varepsilon}(x)= \begin{cases}1, & x \leq 0 \\
0, & x \geq \varepsilon .\end{cases}\right.
$$

For any $\varphi(\tau) \in C_{0}^{\infty}[0, t)$, let $\psi_{\varepsilon}=\bar{\phi}_{\varepsilon}(x, \tau) \varphi(\tau)$ with

$$
\bar{\phi}_{\varepsilon}(x, \tau)=\phi_{2 \varepsilon}\left(x-x_{2}(\tau)\right)-\phi_{1 \varepsilon}\left(x-x_{1}(\tau)\right) .
$$

Then by $(1.4)_{2}$, we have

$$
\iint \psi_{\varepsilon \tau} u+\psi_{\varepsilon x} u^{2}+x \psi_{\varepsilon} d m d t+\int \psi_{\varepsilon}(x, 0) u_{0} \rho_{0} d \eta=0,
$$


which indicates

$$
\begin{aligned}
& \iint \varphi_{\tau} \bar{\phi}_{\varepsilon} u+x \psi_{\varepsilon} d m d \tau+\int \psi_{\varepsilon}(x, 0) u_{0} \rho_{0} d \eta \\
= & -\int_{0}^{t} \int_{x_{1}(\tau)-\varepsilon}^{x_{1}(\tau)} \varphi \phi_{1 \varepsilon}^{\prime}\left(x_{1}^{\prime}(\tau)-u\right) u d m d \tau \\
& +\int_{0}^{t} \int_{x_{2}(\tau)}^{x_{2}(\tau)+\varepsilon} \varphi \phi_{2 \varepsilon}^{\prime}\left(x_{2}^{\prime}(\tau)-u\right) u d m d \tau .
\end{aligned}
$$

By Lemma 4.1 (3) and the fact that $x_{1}, x_{2} \in C_{t}$, we have for almost every $0<\tau<t$,

$$
u\left(x_{i}(\tau)-0, \tau\right)=x_{i}^{\prime}(\tau)=u\left(x_{i}(\tau)+0, \tau\right), \quad i=1,2 .
$$

Thus the right-hand side of (4.11) tends to zero when $\varepsilon$ tends to zero. Letting $\varepsilon \rightarrow 0$ in (4.11) gives

$$
\int_{0}^{t} \int_{x_{1}(\tau)-0}^{x_{2}(\tau)+0} \varphi_{\tau} u+x \varphi d m d \tau+\int_{\xi_{1}}^{\xi_{2}} \varphi(0) u_{0} \rho_{0} d \eta=0 .
$$

For any $0 \leq s<t$, we choose $0<\delta<t-s$. Let $\varphi(\tau)=\varphi_{\delta}(\tau)$ satisfy

$$
\varphi_{\delta}(\tau)= \begin{cases}1, & 0 \leq \tau \leq s \\ 0, & \tau \geq s+\delta\end{cases}
$$

Then substituting (4.13) into (4.12) and letting $\delta \rightarrow 0$ yields

$$
\int_{\xi_{1}}^{\xi_{2}} u_{0} \rho_{0} d \eta-\int_{\xi_{1}}^{\xi_{2}} X^{\prime}(\eta, s) \rho_{0}(\eta) d \eta+\int_{0}^{s} \int_{\xi_{1}}^{\xi_{2}} X(\eta, \tau) \rho_{0}(\eta) d \eta d \tau=0
$$

where we have used the fact that for any measurable function $f$ with respect to $m(x, s)_{x}$,

$$
\int_{x_{1}(s)-0}^{x_{2}(s)+0} f(x) d m(x, s)=\int_{\xi_{1}}^{\xi_{2}} f(X(\eta, s)) \rho_{0}(\eta) d \eta
$$

due to Lemma $4.2, \xi\left(x_{1}(\tau), \tau\right)=\xi_{1}$ and $\xi\left(x_{2}(\tau), \tau\right)=\xi_{2}$ for any $0 \leq \tau \leq t$. Let

$$
h(s)=\int_{0}^{s} \int_{\xi_{1}}^{\xi_{2}} X(\eta, \tau) \rho_{0}(\eta) d \eta d \tau .
$$

Then we have from (4.14)

$$
\int_{\xi_{1}}^{\xi_{2}} u_{0} \rho_{0} d \eta-h^{\prime \prime}(s)+h(s)=0, \quad h(0)=0, \quad h^{\prime}(0)=\int_{\xi_{1}}^{\xi_{2}} \eta \rho_{0}(\eta) d \eta .
$$

Direct computation on (4.17) gives that for any $0 \leq s \leq t$,

$$
\begin{aligned}
h^{\prime}(s) & =\int_{\xi_{1}}^{\xi_{2}} \rho_{0}(\eta) X(\eta, s) d \eta=\int_{\xi_{1}}^{\xi_{2}} \rho_{0}(\eta)\left[\eta \operatorname{ch} s+u_{0}(\eta) \operatorname{sh} s\right] d \eta \\
h^{\prime \prime}(s) & =\int_{\xi_{1}}^{\xi_{2}} \rho_{0}(\eta) X^{\prime}(\eta, s) d \eta=\int_{\xi_{1}}^{\xi_{2}} \rho_{0}(\eta)\left[\eta \operatorname{sh} s+u_{0}(\eta) \operatorname{ch} s\right] d \eta
\end{aligned}
$$

Furthermore, we have 
Lemma 4.3. For any $x_{1}<x_{2}$, let $\xi_{1}=\xi\left(x_{1}, t\right)$ and $\xi_{2}=\xi\left(x_{2}, t\right)$; then for any $0 \leq s \leq t$,

$$
\begin{aligned}
\int_{\xi_{1}}^{\xi_{2}} \rho_{0}(\eta) X(\eta, s) d \eta & =\int_{\xi_{1}}^{\xi_{2}} \rho_{0}(\eta)\left[\eta \operatorname{ch} s+u_{0}(\eta) \operatorname{sh} s\right] d \eta \\
\int_{\xi_{1}}^{\xi_{2}} \rho_{0}(\eta) X^{\prime}(\eta, s) d \eta & =\int_{\xi_{1}}^{\xi_{2}} \rho_{0}(\eta)\left[\eta \operatorname{sh} s+u_{0}(\eta) \operatorname{ch} s\right] d \eta .
\end{aligned}
$$

Proof. Without loss of generality, we assume that $x_{1} \in R / C_{t}$ and $x_{2} \in C_{t}$. There are two subcases.

i) $x_{1}=X\left(\xi_{1}-0, t\right)$ : Let

$$
V_{t}=\{x ; \exists \xi \text { s.t. } X(\xi-0, t)<x<X(\xi+0, t)\} .
$$

It is easy to see that for any $x \in V(t)$,

$$
\rho(x, t)=m_{x}(x, t)=0
$$

due to Lemma 4.2. By the definition of $C_{t}$ and $V_{t}, R /\left(C_{t} \cup V_{t}\right)$ is a zero measure set. Thus in this subcase, there exists a sequence of $x_{1 n} \in C_{t}$ satisfying $x_{1 n}<x_{1}$ and $x_{1 n} \rightarrow x_{1}$ as $n \rightarrow \infty$. Set $\xi_{1 n}=\xi\left(x_{1 n}, t\right)$; then the equality (4.18) yields

$$
\int_{\xi_{1 n}}^{\xi_{2}} \rho_{0}(\eta) X(\eta, s) d \eta=\int_{\xi_{1 n}}^{\xi_{2}} \rho_{0}(\eta)\left[\eta \operatorname{ch} s+u_{0}(\eta) \operatorname{sh} s\right] d \eta .
$$

Let $n \rightarrow \infty$; we obtain (4.20) due to Lemma 4.1 (4). The equality (4.21) can be obtained in the same way.

ii) $X\left(\xi_{1}-0, t\right)<x_{1} \leq X\left(\xi_{1}+0, t\right)$ : Let $\bar{x}_{1}=X\left(\xi_{1}+0, t\right)$. Then we have $\xi\left(\bar{x}_{1}, t\right)=$ $\xi\left(x_{1}, t\right)=\xi_{1}$. Following the same argument as i), we obtain (4.20) and (4.21). Hence Lemma 4.3 is proved.

Now we define

$$
\begin{aligned}
& q(x, t)=\int_{0}^{\xi(x, t)} \rho_{0}(\eta)\left[\eta \operatorname{sh} t+u_{0}(\eta) \operatorname{ch} t\right] d \eta \\
& n(x, t)=\int_{0}^{\xi(x, t)} \rho_{0}(\eta)\left[\eta \operatorname{ch} t+u_{0}(\eta) \operatorname{sh} t\right] d \eta .
\end{aligned}
$$

We have

LEMmA 4.4. $q_{x}=u(x, t) m_{x}$ and $n_{x}=x m_{x}$ hold in the sense of Radon-Nykodym in $x$.

Proof. Let $\Gamma_{t}=\{x ;(x, t) \in \Gamma\}$ and $A=C_{t} \cup V_{t} \cup \Gamma_{t}$. Then by the definition, $R / A$ consists of at most countable points where $m(x, t)$ is continuous. It is noted that $\rho$ is a vacuum in $V_{t}$. Therefore we only need to show that Lemma 4.4 holds in the set $\Gamma_{t}$ everywhere and the set $C_{t}$ almost everywhere. Let $x \in C_{t}$ satisfy

$$
\lim _{\varepsilon \rightarrow 0} \frac{\int_{\xi-\varepsilon}^{\xi+\varepsilon} \rho_{0} u_{0} d \eta}{\int_{\xi-\varepsilon}^{\xi+\varepsilon} \rho_{0} d \eta}=u_{0}(\xi), \quad \xi=\xi(x, t) .
$$

It is easy to check that such points are dense in $C_{t}$. Then there exist two sequences of $x_{1 n}, x_{2 n} \in C_{t}$ satisfying $x \in\left(x_{1 n}, x_{2 n}\right), x_{1 n} \rightarrow x_{1}$ and $x_{2 n} \rightarrow x_{2}$. From Lemma 4.3, we 
have

$$
\int_{\xi\left(x_{1 n}, t\right)}^{\xi\left(x_{2 n}, t\right)} \rho_{0}(\eta) X^{\prime}(\eta, s) d \eta=\int_{\xi\left(x_{1 n}, t\right)}^{\xi\left(x_{2 n}, t\right)} \rho_{0}(\eta)\left[\eta \operatorname{sh} s+u_{0}(\eta) \operatorname{ch} s\right] d \eta
$$

Letting $n \rightarrow \infty$ yields

$$
X^{\prime}(\xi, s)=\xi \operatorname{sh} s+u_{0}(\xi) \operatorname{ch} s, 0 \leq s \leq t .
$$

Let $s=t$. We have

$$
q_{x}=u(x, t) m_{x}, \quad u(x, t)=X^{\prime}(\xi, t)=\xi \operatorname{sh} t+u_{0}(\xi) \operatorname{ch} t .
$$

Using the same argument, we obtain that for any $x \in \Gamma_{t}$ and $0 \leq s \leq t$,

$$
\int_{\xi(x, t)}^{\eta(x, t)} \rho_{0}(\eta) X^{\prime}(\eta, s) d \eta=\int_{\xi(x, t)}^{\eta(x, t)} \rho_{0}(\eta)\left[\eta \operatorname{sh} s+u_{0}(\eta) \operatorname{ch} s\right] d \eta .
$$

Let $s=t$. Then $X^{\prime}(\eta, t)=u(x, t)=\int_{\xi(x, t)}^{\eta(x, t)} \rho_{0}\left[\eta \operatorname{sh} t+u_{0} \operatorname{ch} t\right] d \eta$ holds for any $\eta \in$ $(\xi(x, t), \eta(x, t))$ due to Lemma 4.1. Hence we obtain $q_{x}=u(x, t) m_{x}$. The relation $n_{x}=x m_{x}$ can be treated in the same way. Therefore, the proof of Lemma 4.4 is completed.

In terms of Lemma 4.4, Eq. (4.1) yields

$$
m_{t}+q_{x}=0 \text {. }
$$

It is noted that Eq. (4.31) is conserved and $m$ and $q$ are left continuous functions. We introduce the following generalized potential $\Phi(x, t)$

$$
\Phi(x, t)=\oint_{(0,0)}^{(x, t)} m(x, t) d x-q(x, t) d t .
$$

Obviously, the potential $\Phi(x, t)$ is independent of the integral path and $\Phi_{x}=m, \Phi_{t}=-q$ hold in the sense of distributions. If we can show

$$
\Phi(x, t)=-\min _{y} F(y ; x, t),
$$

where $F(y ; x, t)$ is introduced in $(1.10)$, then it is natural to imply the uniqueness Theorem 2 because $F(y ; x, t)$ only depends on the initial data. We shall establish (4.33) by two integral paths.

For any point $\left(x_{0}, t_{0}\right) \in R_{+}^{2}$, there exists at least one curve $L^{\prime}$ through $\left(x_{0}, t_{0}\right)$ and $\left(\xi\left(x_{0}, t_{0}\right), 0\right)$ such that $\xi(x, t)$ keeps constant along $L^{\prime}$ due to Lemma $4.1(5)$. The first integral path is chosen from $(0,0)$ to $\left(\xi\left(x_{0}, t_{0}\right), 0\right)$ along the $x$-axis and from $\left(\xi\left(x_{0}, t_{0}\right), 0\right)$ to $\left(x_{0}, t_{0}\right)$ along the curve $L^{\prime}$. Let $\xi_{0}=\xi\left(x_{0}, t_{0}\right)$. We compute

$$
\begin{aligned}
\Phi\left(x_{0}, t_{0}\right)= & \int_{0}^{\xi_{0}} m_{0}(\eta) d \eta+\int_{L^{\prime}} m(x, t) d x-q(x, t) d t \\
= & \int_{0}^{\xi_{0}}\left(\xi_{0}-\eta\right) \rho_{0} d \eta-\int_{0}^{t_{0}} \int_{0}^{\xi_{0}}\left[\eta \operatorname{sh} s+u_{0}(\eta) \operatorname{ch} s\right] \rho_{0} d \eta d s \\
& \quad+\left(x_{0}-\xi_{0}\right) \int_{0}^{\xi_{0}} \rho_{0} d \eta \\
= & \int_{0}^{\xi_{0}} \rho_{0}\left[x_{0}-\left(\eta \operatorname{cht} t_{0}+u_{0} \operatorname{sh} t_{0}\right)\right] d \eta
\end{aligned}
$$


On the other hand, we choose another integral path which is from $(0,0)$ to $\left(y_{0}, 0\right)$ along the $x$-axis and from $\left(y_{0} .0\right)$ to $\left(x_{0}, t_{0}\right)$ along the curve $L: x=x(t)=y_{0} \operatorname{ch} t+v_{0} \operatorname{sh} t$, $0 \leq t \leq t_{0}$, where $v_{0}=\frac{1}{\operatorname{sh} t_{0}}\left(x_{0}-y_{0} \operatorname{cht} t_{0}\right)$. Here $y_{0}$ is any constant. We compute

$$
\begin{aligned}
\Phi\left(x_{0}, t_{0}\right) & =\int_{0}^{y_{0}} m_{0}(\eta) d \eta+\int_{L} m d x-q d t \\
& =\int_{0}^{y_{0}} m_{0}(\eta) d \eta+\int_{0}^{t_{0}} x^{\prime}(t) m(x(t), t)-q(x(t), t) d t .
\end{aligned}
$$

To calculate the last term of (4.35), we use the following lemma, whose proof will be given at the end of this section.

LEMmA 4.5. Let $x(\tau)=y_{0} \operatorname{ch} \tau+v_{0} \operatorname{sh} \tau$ and $y(\tau)=\xi(x(\tau), \tau), 0 \leq \tau \leq t$. Then for any $0<\tau<t$,

$$
\int_{y_{0}}^{y(\tau)} \rho_{0}(\eta) d \eta x^{\prime}(\tau) \geq \int_{y_{0}}^{y(\tau)} \rho_{0}(\eta)\left[\eta \operatorname{sh} \tau+u_{0}(\eta) \operatorname{ch} \tau\right] d \eta .
$$

In terms of Lemma 4.5, we obtain

$$
\begin{aligned}
x^{\prime}(t) m(x(t), t)-q(x(t), t) & =\int_{0}^{y(t)} \rho_{0}\left(x^{\prime}(t)-\eta \operatorname{sh} t-u_{0} \operatorname{ch} t\right) d \eta \\
& \geq \int_{0}^{y_{0}} \rho_{0}\left(x^{\prime}(t)-\eta \operatorname{sh} t-u_{0} \operatorname{ch} t\right) d \eta
\end{aligned}
$$

Substituting (4.37) into (4.35) implies

$$
\begin{aligned}
\Phi\left(x_{0}, t_{0}\right) & \geq \int_{0}^{y_{0}} \rho_{0}\left(y_{0}-\eta\right) d \eta+\int_{0}^{y_{0}} \int_{0}^{t_{0}} \rho_{0}\left[\eta \operatorname{sh} t+u_{0} \operatorname{ch} t\right] d \eta \\
& =\int_{0}^{y_{0}} \rho_{0}\left(x_{0}-\eta \operatorname{ch} t_{0}-u_{0} \operatorname{sh} t_{0}\right) d \eta .
\end{aligned}
$$

Since $y_{0}$ is arbitrary, we have

$$
\Phi\left(x_{0}, t_{0}\right) \geq \max _{y} \int_{0}^{y} \rho_{0}\left(x_{0}-\eta \operatorname{ch} t_{0}-u_{0} \operatorname{sh} t_{0}\right) d \eta .
$$

Combining (4.35) and (4.39), we obtain

$$
\begin{aligned}
\Phi\left(x_{0}, t_{0}\right) & =\max _{y} \int_{0}^{y} \rho_{0}\left(x_{0}-\eta \operatorname{ch} t_{0}-u_{0} \operatorname{sh} t_{0}\right) d \eta, \\
& =-\min _{y} F\left(y ; x_{0}, t_{0}\right) .
\end{aligned}
$$

On the other hand, by the existence Theorem 1 , we can construct a standard entropy solution $m_{s}, u_{s}$ satisfying $m_{s}=\Phi_{x}, q_{s}=-\Phi_{t}$, where $\left(q_{s}\right)_{x}=u_{s}\left(m_{s}\right)_{x}$. Thus by (4.40) we have $m=m_{s}, q=q_{s}$ a.e. in $t>0$. Therefore the uniqueness Theorem 2 is proved.

Proof of Lemma 4.5. Without loss of generality, we assume that $y_{0}<x(\tau)$. Then we have $X\left(y_{0}, \tau\right)<x(\tau)$. We divide our proof into two subcases.

i) For any $0<s \leq \tau, X\left(y_{0}, s\right)<x(s)$. Let

$$
L P=\left\{\xi \in R ; \lim _{\varepsilon \rightarrow 0} \frac{\int_{\xi-\varepsilon}^{\xi+\varepsilon} \rho_{0} u_{0} d \eta}{\int_{\xi-\varepsilon}^{\xi+\varepsilon} \rho_{0} d \eta}=u_{0}(\xi)\right\} .
$$


Obviously, the set LP is dense in R. For any $y \in\left(y_{0}, y(\tau)\right) \cap L P / U$, where $U$ is introduced in Lemma $4.1(3)$, the curve $X(y, s)$ must intersect with the curve $\mathrm{L}: x=x(s)$ at some time $0<t(y)<\tau$. See Fig 4.1. Let

$$
\xi(y)=\lim _{s \rightarrow t(y)} \xi(X(y, s), s), \quad \eta(y)=\lim _{s \rightarrow t(y)} \eta(X(y, s), s) .
$$

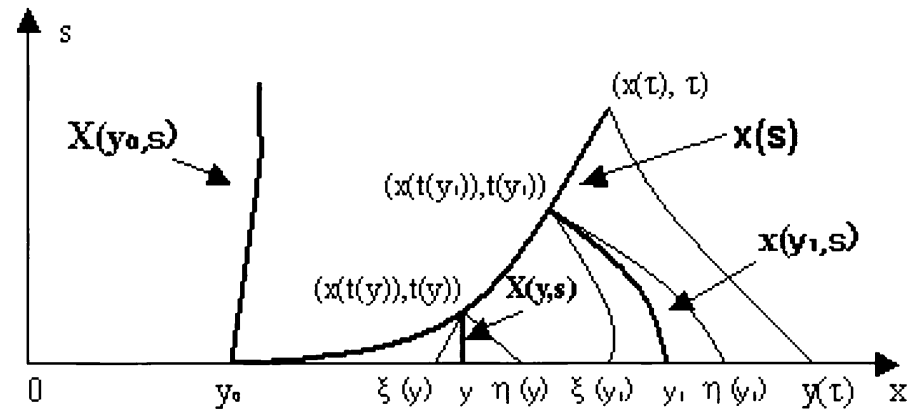

Fig 4.1

Then we have

$$
\xi(y) \leq y \leq \eta(y) .
$$

If $\xi(y)=y=\eta(y)$, equality (4.28) implies

$$
X(y, s)=y \operatorname{ch} s+u_{0}(y) \operatorname{sh} s, \quad 0<s<t(y) .
$$

Since $y_{0}<y$ and

$$
x(t(y))=y_{0} \operatorname{ch} t(y)+u_{0}(y) \operatorname{sh} t(y)=X(y, t(y))=y \operatorname{ch} t(y)+u_{0}(y) \operatorname{sh} t(y),
$$

we obtain $u_{0}(y)<v_{0}$. A simple calculation shows that

$$
\frac{y-y_{0}}{v_{0}-u_{0}(y)}=\frac{\operatorname{sh} t(y)}{\operatorname{ch} t(y)} \leq \frac{\operatorname{ch} \tau}{\operatorname{sh} \tau}
$$

which indicates

$$
x^{\prime}(\tau) \geq y \operatorname{sh} \tau+u_{0}(y) \operatorname{ch} \tau .
$$

If $\xi(y) \neq \eta(y)$, let $I_{y}=(\xi(y), \eta(y))$. From Lemma 4.4, we have

$$
\begin{aligned}
& \lim _{s \rightarrow t(y)} X^{\prime}(y, s) \int_{\xi(y)}^{\eta(y)} \rho_{0}(\eta) d \eta=\int_{\xi(y)}^{\eta(y)} \rho_{0}(\eta)\left[\eta \operatorname{sh} s+u_{0}(\eta) \operatorname{ch} s\right] d \eta \\
& \lim _{s \rightarrow t(y)} X(y, s) \int_{\xi(y)}^{\eta(y)} \rho_{0}(\eta) d \eta=\int_{\xi(y)}^{\eta(y)} \rho_{0}(\eta)\left[\eta \operatorname{ch} s+u_{0}(\eta) \operatorname{sh} s\right] d \eta
\end{aligned}
$$

Noting that $\lim _{s \rightarrow t(y)} X(y, s)=x(t(y))$ and $\lim _{s \rightarrow t(y)} X^{\prime}(y, s)<x^{\prime}(t(y))$, we get

$$
y_{0} \int_{\xi(y)}^{\eta(y)} \rho_{0} d \eta<\int_{\xi(y)}^{\eta(y)} \rho_{0} \eta d \eta
$$


and

$$
v_{0} \int_{\xi(y)}^{\eta(y)} \rho_{0} d \eta>\int_{\xi(y)}^{\eta(y)} \rho_{0} u_{0} d \eta
$$

Applying the same argument as in (4.46) and (4.47) yields

$$
X^{\prime}(\tau) \int_{\xi(y)}^{\eta(y)} \rho_{0}(\eta) d \eta \geq \int_{\xi(y)}^{\eta(y)} \rho_{0}(\eta)\left[\eta \operatorname{sh} \tau+u_{0}(\eta) \operatorname{ch} \tau\right] d \eta .
$$

On the other hand, it is easy to check that, for all of these intervals, $I_{y}$ either is equivalent or disjoint to each other due to Lemma 4.1 (1) and (2). Let $\Delta=\left\{y ;\left|I_{y}\right| \neq\right.$ $\left.0, y \in\left(y_{0}, y(\tau)\right)\right\}$; then $\Delta$ consists of at most denumerable open intervals which disjoint each other. Thus by (4.50), we have

$$
X^{\prime}(\tau) \int_{\Delta} \rho_{0}(\eta) d \eta \geq \int_{\Delta} \rho_{0}(\eta)\left[\eta \operatorname{sh} \tau+u_{0}(\eta) \operatorname{ch} \tau\right] d \eta .
$$

Since (4.47) holds for any $y \in\left(y_{0}, y(\tau)\right) \cap L P /(\Delta \cup U)$, we have

$$
X^{\prime}(\tau) \int_{\left(y_{0}, y(\tau)\right) / \Delta} \rho_{0}(\eta) d \eta \geq \int_{\left(y_{0}, y(\tau)\right) / \Delta} \rho_{0}(\eta)\left[\eta \operatorname{sh} \tau+u_{0}(\eta) \operatorname{ch} \tau\right] d \eta .
$$

Combining (4.51) and (4.52) implies (4.36).

ii) $\exists 0<s_{0} \leq \tau$, s.t. $X\left(y_{0}, s_{0}\right)=x\left(s_{0}\right)$.

Suppose that there exists $n(n \geq 2)$ intersection points between $X\left(y_{0}, s\right)$ and $x(s)$, $0 \leq s<\tau$. We denote these points by $\left(x_{i}, s_{i}\right), i=1,2, \cdots, n$ with $s_{n}=0<s_{n-1}<\cdots<$ $s_{1} \leq \tau$. See Fig 4.2. Let $\xi_{i}=\xi\left(x_{i}, s_{i}\right), \eta_{i}=\eta\left(x_{i}, s_{i}\right), i=1, \cdots, n-1$ and $\xi_{n}=\eta_{n}=y_{0}$ and $\eta_{0}=y(\tau)$.

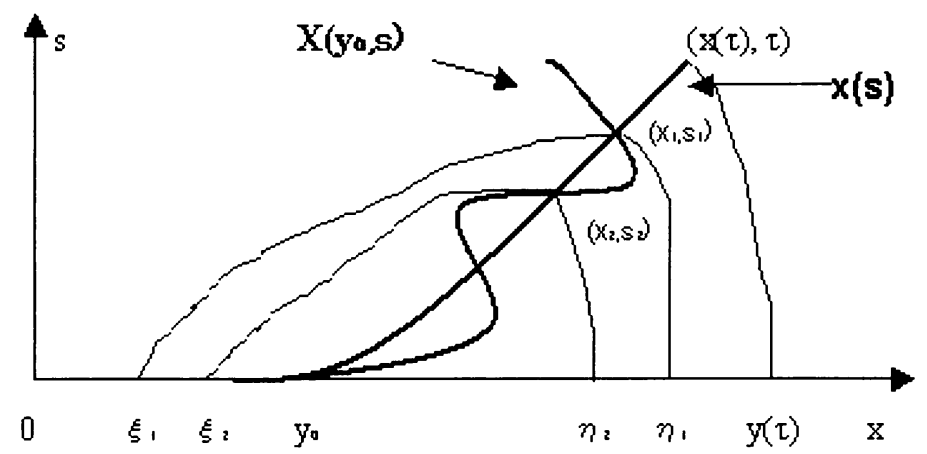

Fig 4.2

It is easy to check that $\left[y_{0}, y(\tau)\right]=\sum_{i=0}^{n-1}\left[\eta_{i+1}, \eta_{i}\right]$. It is observed that when $s_{1}<$ $s \leq \tau, X\left(y_{0}, s\right)<x(s)$ holds. Using the same argument as $\left.\mathrm{i}\right)$, we have

$$
X^{\prime}(\tau) \int_{\eta_{1}}^{y(\tau)} \rho_{0}(\eta) d \eta \geq \int_{\eta_{1}}^{y(\tau)} \rho_{0}(\eta)\left[\eta \operatorname{sh} \tau+u_{0}(\eta) \operatorname{ch} \tau\right] d \eta
$$


When $s_{2}<s \leq s_{1}$, we have $x(s)<X\left(y_{0}, s\right)$. Again using the same argument as i) yields

$$
X^{\prime}(\tau) \int_{\eta_{2}}^{\eta_{1}} \rho_{0}(\eta) d \eta \leq \int_{\eta_{2}}^{\eta_{1}} \rho_{0}(\eta)\left[\eta \operatorname{sh} \tau+u_{0}(\eta) \operatorname{ch} \tau\right] d \eta
$$

We note that

$$
x^{\prime}\left(s_{1}\right) \int_{\xi_{1}}^{\eta_{1}} \rho_{0}(\eta) d \eta \geq X^{\prime}\left(y_{0}, s_{1}\right) \int_{\xi_{1}}^{\eta_{1}} \rho_{0}(\eta) d \eta=\int_{\xi_{1}}^{\eta_{1}} \rho_{0}(\eta)\left[\eta \operatorname{sh} s_{1}+u_{0}(\eta) \operatorname{ch} s_{1}\right] d \eta
$$

which indicates

$$
X^{\prime}(\tau) \int_{\xi_{1}}^{\eta_{1}} \rho_{0}(\eta) d \eta \geq \int_{\xi_{1}}^{\eta_{1}} \rho_{0}(\eta)\left[\eta \operatorname{sh} \tau+u_{0}(\eta) \operatorname{ch} \tau\right] d \eta
$$

We also note that

$$
X^{\prime}(\tau) \int_{\xi_{2}}^{\eta_{2}} \rho_{0}(\eta) d \eta \leq \int_{\xi_{2}}^{\eta_{2}} \rho_{0}(\eta)\left[\eta \operatorname{sh} \tau+u_{0}(\eta) \operatorname{ch} \tau\right] d \eta
$$

Thus we obtain

$$
X^{\prime}(\tau) \int_{\left(\xi_{1}, \xi_{2}\right) \cup\left(\eta_{2}, \eta_{1}\right)} \rho_{0}(\eta) d \eta \geq \int_{\left(\xi_{1}, \xi_{2}\right) \cup\left(\eta_{2}, \eta_{1}\right)} \rho_{0}(\eta)\left[\eta \operatorname{sh} \tau+u_{0}(\eta) \operatorname{ch} \tau\right] d \eta .
$$

Substituting (4.54) into (4.58) implies that

$$
X^{\prime}(\tau) \int_{\eta_{2}}^{\eta_{1}} \rho_{0}(\eta) d \eta \geq \int_{\eta_{2}}^{\eta_{1}} \rho_{0}(\eta)\left[\eta \operatorname{sh} \tau+u_{0}(\eta) \operatorname{ch} \tau\right] d \eta
$$

In the same way, we can show for any $i=0, \cdots, n-1$,

$$
X^{\prime}(\tau) \int_{\eta_{i+1}}^{\eta_{i}} \rho_{0}(\eta) d \eta \geq \int_{\eta_{i+1}}^{\eta_{i}} \rho_{0}(\eta)\left[\eta \operatorname{sh} \tau+u_{0}(\eta) \operatorname{ch} \tau\right] d \eta
$$

Adding all of these inequalities yields

$$
X^{\prime}(\tau) \int_{y_{0}}^{y(\tau)} \rho_{0}(\eta) d \eta \geq \int_{y_{0}}^{y(\tau)} \rho_{0}(\eta)\left[\eta \operatorname{sh} \tau+u_{0}(\eta) \operatorname{ch} \tau\right] d \eta .
$$

Therefore Lemma 4.5 is proved.

Acknowledgment. The authors wish to thank Prof. Xiaqi Ding for kindly suggesting this problem and for many helpful discussions.

\section{REFERENCES}

[1] F. Bouchut, On zero pressure gas dynamics, Advances in kinetic theory and computing, Series on Advances in Mathematics for Applied Sciences, World Scientific, Vol.22: 171-190 (1994).

[2] F. Bouchut and F. James, One-dimensional transportation equations with discontinuous coefficients, Nonlinear Analysis TMA, 32 (1998), 891-933.

[3] F. Bouchut and F. James, Duality solutions for pressureless gases, monotone scalar conservation laws, and uniqueness, Comm. Part. Diff. Equs., 24 (1999), 2173-2190.

[4] Y. Brenier and E. Grenier, Sticky particles and scalar conservation laws, SIAM J. Num. Analysis, Vol. 35, No. 6, 1998, 2317-2328.

[5] C. Dafermos, Generalized characteristics and the structure of solutions of hyperbolic conservation laws, Indiana Univ. Math. Journal, Vol. 26, No. 6 (1977): 1097-1119.

[6] G. Dal Maso, P. Le Floch, and F. Murat, Definition and weak stability of nonconservative products, J. Math. Pure Appl. 74 (1995), 483-548.

[7] X. Ding, Q. Jiu, and C. He, On a nonhomogeneous Burger's equation, Sci. China Ser. A, 44 (2001), No. $8984-993$. 
[8] X. Ding and Y. Ding, Viscosity method of a non-homogeneous Burgers equation, 2002, preprint.

[9] W. E, Y. Rykov, and Y. Sinai, Generalized variational principles, global weak solutions and behavior with random initial data for systems of conservation laws arising in adhesion particle dynamics, Comm. Math. Phys., 177 (1996), 349-380.

[10] E. Hopf, The partial differential equation $u_{t}+u u_{x}=\mu u_{x x}$, Comm. Pure Appl. Math., 1950, 3: 201-230.

[11] F. Huang and Z. Wang, Well posedness for pressureless flow, Comm. Math. Phys. 222 (2001), no. 1, 117-146.

[12] S. Chen, J. Li, and T. Zhang, Explicit construction of measure solutions of the Cauchy problem for the transportation equations, Science in China (Series A), Vol. 40, 12: 1287-1299(1997).

[13] O. Oleinik, Discontinuous solutions of nonlinear differential equations, Amer. Math. Soc. Transl. (2), 26 (1963), 95-172.

[14] F. Poupaud and M. Rascle, Measure solutions to the linear multidimensional transportation with discontinuous coefficients, Comm. Part. Diff. Equs., 22 (1997), 337-358.

[15] Z. Wang, F. Huang, and X. Ding, On the Cauchy problem of transportation equation, Acta Math. Appl. Sinica, No. 2, 1997, 113-122.

[16] Z. Wang and X. Ding, Uniqueness of generalized solution for the Cauchy problem of transportation equations, Acta Math. Scientia 17 (1997), n. 3, 341-352. 